Toronto, Canada. Case and control schools were defined as those with the highest and lowest quartile of PMVC rates, calculated using census data. Potential risk factors included built and nonbuilt environment variables obtained from municipal data sources as well as via direct observational counts done in the spring, 2015, to measure the proportion of children walking to school. Logistic regression was used to compare case versus control schools stratified by geographic location (downtown vs inner suburbs).

Results The mean PMVC rate in case schools $(\mathrm{n}=50)$ was $13.4 / 10,000 /$ year and in controls $(n=50)$ was $1.75 / 10,000 /$ year. Walking was not associated with high PMVC rates after adjustment for the built environment and school social disadvantage. Overall, lower residential (OR 0.56, 95\% CI: 0.37, 0.86) and higher one-way street densities (OR 4.00, 95\% CI: 1.76, 9.08), school crossing guards (OR 3.65, 95\% CI: 1.10, 12.20) and higher social disadvantage (OR 1.37, 95\% CI: 1.11, 1.70) were associated with high PMVC schools, Similar associations of high PMVC schools with built environment features were found in the inner suburbs; however, there was a stronger association with school social disadvantage downtown.

Conclusions Walking to school was unrelated to high PMVC rates after controlling for the built environment. The built environment and school disadvantage were associated with higher PMVC rates with possible differences by geographic location.

\section{A SYSTEMATIC REVIEW AND META-ANALYSIS OF SCHOOL BASED PROGRAMMES TO PREVENT CHILDHOOD INJURIES}

Elizabeth Orton, Jacqueline Mhizha-Murira, Mandy Clarkson, Jessica Whitehead, Michael Craig Watson, Caroline A Mulvaney, Joy Staniforth, Munish Bhuchar, Denise Kendrick. University of Nottingham, United Kingdom

\subsection{6/injuryprev-2016-042156.404}

Background The aim of this Cochrane systematic review was to evaluate the effectiveness and cost-effectiveness of school-based education programmes to prevent unintentional injuries in children and young people.

Methods A total of 28 electronic databases and websites were searched. We included randomised and non-randomised controlled trials and controlled before-and-after studies of primary and secondary prevention interventions, delivered in the school setting, aimed at a range of injury mechanisms. The primary outcome was self-reported or medically-attended unintentional (or unspecified) injuries and secondary outcomes were observed safety skills, observed behaviour, self-reported behaviour and safety practices, safety knowledge and health economic outcomes.

Results 27 studies reported in 29 articles were included. Interventions comprised information giving, peer education or were multi-component. 7 studies reported the primary outcome of injury occurrence and only 3 of these were similar enough to combine in a meta-analysis with a pooled incidence rate ratio of 0.76 (95\% CI: $0.49,1.17)$ and significant heterogeneity between effect sizes $\left(\mathrm{Chi}^{2}=10.38, \mathrm{df}=2, \mathrm{P}=0.006 \mathrm{I}^{2}=81 \%\right)$. Safety skills reported in 2 studies showed significant improvement, as did all 4 studies reporting observed safety behaviours and 13 out of 19 studies describing self-reported behaviour. The 21 studies measuring changes in safety knowledge were varied in their focus, including water, burn, sport, brain and spinal cord, agricultural or mixed injury prevention programmes and most reported that safety knowledge improved. Only one study reported intervention costs but did not undertake a full economic evaluation.

Conclusions There is good evidence that school-based injury prevention programmes improve safety skills, behaviour and knowledge. We found insufficient economic studies to assess cost-effectiveness.

\section{PREVENTING HOME INJURIES AMONG CHILDREN IN MALAYSIA: A CLUSTER RANDOMISED CONTROLLED TRIAL}

${ }^{1}$ Abdulgafoor M Bachani, ${ }^{1}$ Xiaoge Julia Zhang, ${ }^{2}$ Kulanthayan Mani, ${ }^{1}$ Adnan A Hyder. 'Johns Hopkins International Injury Research Unit, Johns Hopkins University Bloomberg School of Public Health, USA; ${ }^{2}$ Universiti Putra Malaysia, Serdang, Malaysia

\subsection{6/injuryprev-2016-042156.405}

Background Child injury at home has become a growing concern in developing countries. This project aims to evaluate the effectiveness of two intervention strategies-home-based safety tutorial program and educational pamphlet - to reduce in-home hazards for unintentional child injuries within the home in Malaysia.

Methods We conducted a prospective cluster randomised controlled trial in Hulu Langat district, Malaysia. We randomised 59 clusters to two study arms (30 for tutorial and 29 for pamphlet) with 30 households per cluster. On an initial household visit, a baseline home safety hazard assessment was conducted, followed by the intervention and two follow-up visits at 2 and 4 months. The outcome measures are in-home hazards for child injuries, and incidence of child home injuries.

Results The study enrolled 1170 households, with 13324 children 1-5 years of age. Overall, almost 40\% (5061) of children experienced an injury at home in the 3 months preceding our initial visit. The three most common types of injuries reported were falls $(86.2 \%)$, poisoning $(2.9 \%)$, and animal bites $(2.1 \%)$. The most common types of safety hazards for such injuries observed were having pedestal fan within reach of children in living/sleeping area (tutorial: $45 \%$ vs. pamphlet: $46 \%, \mathrm{p}=0.13$ ), presence of open buckets of water ( $45 \%$ vs. $44 \%$ ), and having lock of the bathroom door within reach of children (41\% vs. $45 \%)$. Adjusting for socio-demographic factors at household level and caregiver characteristics, estimates of a generalised linear model fit showed that presence of open buckets of water significantly predicts child injuries (OR $=1.8,95 \% \mathrm{CI}$ : 1.4-2.4).

Conclusions The study improves understanding of the burden of household injuries among children in a Malaysian district, and findings can guide intervention strategies for addressing home injuries among children. Materials and interventions developed in this study can be adapted to other settings. 


\section{Sports and Exercise Safety, Safety Culture, Older People Safety, Traffic Safety}

\section{Post Mon 1.2}

\section{EPIDEMIOLOGY OF KNEE SPRAINS IN US HIGH SCHOOL AND COLLEGIATE ATHLETICS}

'Lauren A Pierpoint, ${ }^{2}$ Zachary Y Kerr, 'Dustin W Currie, 'Dawn R Comstock. 'Program for Injury Prevention, Education and Research (PIPER), Department of Epidemiology, Colorado School of Public Health, Aurora, CO, USA; ${ }^{2}$ Datalys Centre for Sports Injury Research and Prevention, Indianapolis, IN, USA

\subsection{6/injuryprev-2016-042156.406}

Background Over two million sports-related knee injuries present to US emergency departments annually. Knee injuries frequently require costly surgical repair, and knee sprains are one of the most common injuries in athletes. Thus, understanding injury patterns across the age spectrum is important to identify areas for prevention.

Methods Knee sprain and athlete exposure (AE) data were collected for 20 sports using the High School Reporting Information Online database for high school athletes and the National Collegiate Athletic Association Injury Surveillance Program for college athletes during the 2009/10-2014/15 acdemic years. We report knee sprain rates per 10,000 AEs and rate ratios with 95\% confidence intervals (RR; CI).

Results The knee sprain rate was higher in college (1.03) than high school $(0.35 ; \mathrm{RR}=2.46,95 \% \mathrm{CI}:=2.31-2.61)$. Sports with the highest rates in college were men's wrestling (1.21), men's football (0.66), and women's soccer (0.61); the highest in high school were boys' football (0.35), girls' soccer (0.26), and girls' gymnastics (0.23). In gender-comparable sports, females had higher rates than males (college $\mathrm{RR}=1.70$, 95\% $\mathrm{CI}:=1.40-2.07$; high school $\mathrm{RR}=2.21$, 95\% CI: $=1.97-$ 2.48); college men had higher rates of torn cartilage ( $\mathrm{RR}=4.19$, 95\% CI: $=3.19-5.51)$ and PCL injuries $(\mathrm{RR}=29.51,95 \%$ $\mathrm{CI}:=19.64-44.34)$ than high school boys; and college women had higher rates of ACL (RR $=2.30,95 \%$ CI: $=1.86-2.85)$ and PCL $(\mathrm{RR}=2.99,95 \% \mathrm{CI}:=1.52-5.88)$ injuries than high school girls. A larger percentage of females in college required surgery $(43.0 \%)$ vs. high school (34.9\%). Player contact was the most common injury mechanism across age groups (55.0\% each). Conclusions In gender-comparable sports, females at both age levels had higher knee injury rates than males. College athletes had higher knee injury rates than high school athletes, perhaps due to level of play or biological differences. Both sex and age should be considered when developing targeted injury prevention efforts.

\section{HOW TO SPEAK SO TODAY'S PARENTS WILL LISTEN}

Martha Wilcox, Line Storgaard-Conley. Safe Kids Worldwide, USA;

\subsection{6/injuryprev-2016-042156.407}

Background To help families protect children from injury, we have to reach them in channels they are tuning into using messages that resonate. Today's parents are getting much of their information through channels that didn't exist ten years ago. Safe Kids has undergone a messaging transformation, changing our tone, simplifying our messaging for low-literacy audiences and building new media channels to connect with parents.
We conducted a series of focus groups among mothers and grandmothers. We recruited from two populations: low literacy and average literacy. The topic was medication safety.

We also tested several types of posts on Facebook, tracking engagement.

Objective The objective of this session is to educate injury professionals on strategies for connecting with today's families.

The culture of communications has changed dramatically in the last ten years. As safety experts, we need to evolve how we reach today's families.

Results We learned the best way to present messages to parents so they are intrigued to learn more. We also learned when it helps to include statistics and when parents tune statistics out.

To address channels, we tested several social media strategies to determine which ones deliver the best results. Our success is evident in our growth. In just four years, our Facebook page has grown from 35,000 likes to more than 1,000,000 likes.

Conclusions To impact the culture of safety, we need to understand the culture of communicating to today's parents so we can meet parents where they are. Safe Kids Worldwide is eager to share what we have learned about tone, messaging and communications channels. In conversations at past conferences with injury professionals around the world, we believe this is a topic of universal interest and essential for connecting with families to reduce injuries in children in the future.

\section{IMPLEMENTATION PROJECT ON PREVENTION OF HOME AND LEISURE INJURIES}

${ }^{1}$ Tiina Tikkanen, ${ }^{2}$ Heikki Laurikainen, ${ }^{1}$ Kristiina Myllyrinne. ${ }^{1}$ Finnish Red Cross, Finland; ${ }^{2}$ the Finnish National Rescue Association, Finland

\subsection{6/injuryprev-2016-042156.408}

Background The campaign for the Prevention of Home Injuries started in 1993. As of 2015 the campaign was granted a project funding from Finland's Slot Machine Association. Finnish Red Cross coordinates the project. Partner organisations include The Ministry of Social Affairs and Health, Ministry of the Interior, The Finnish National Rescue Association, Federation of Finnish Financial Services, The Association of Finnish Local and Regional Authorities, The Finnish Swimming Teaching and Lifesaving Federation, National Institute of Health and Welfare, The National Defence Training Association of Finland, SOSTE Finnish Federation for Social Affairs and Health and Finnish Association for Substance Abuse Prevention.

Description of the problem The amount of home and leisure accidents in Finland is high. During the year 2013, 2548 people (out of 5.4 million inhabitants) died in injuries. 2268 victims died in home and leisure injuries, 259 in traffic accidents and 22 in work place accidents. The most common type of accident is falling or tumbling. Home and leisure accident prevention is challenging because it extends to the area of private life and it is not clearly legislated.

Results Collaboration between stakeholders in accident prevention is ensured by the project. The project's campaigns are implemented together with traffic and occupational accident injuries prevention stakeholders to strengthen the link between the safety cultures in different environments. The project delivers information to citizens through campaigns, media and the web and by people who are employed or volunteer in the social, health, educational or rescue sector. Non-governmental organisations are 